\title{
Clinical Usefulness of 18F-fluorodeoxyglucose Positron Emission Tomography for Enthesitis-related Arthritis Diagnosis
}

NATSUMI INOUE, MD, Department of Pediatrics, School of Medicine, Institute of Medical, Pharmaceutical, and Health Sciences, Kanazawa University; MAO MIZUTA, MD, Department of Pediatrics, School of Medicine, Institute of Medical, Pharmaceutical, and Health Sciences, Kanazawa University; MASAKI SHIMIZU, MD, PhD, Department of Pediatrics, School of Medicine, Institute of Medical, Pharmaceutical, and Health Sciences, Kanazawa University, Kanazawa, Japan. Address correspondence to Dr. M. Shimizu, Department of Pediatrics, School of Medicine, Institute of Medical, Pharmaceutical, and Health Sciences, Kanazawa University, 13-1 Takaramachi, Kanazawa, 920-8641, Japan. E-mail: shimizum@ staff.kanazawa-u.ac.jp. J Rheumatol 2016;43:1434-5; doi:10.3899/jrheum.160047

Enthesitis-related arthritis (ERA) diagnosis is sometimes challenging because the frequency of back pain is lesser and that of peripheral symptoms is higher compared with adult spondyloarthritis ${ }^{1}$. Distinguishing ERA from other subtypes of juvenile idiopathic arthritis is also sometimes difficult when inappropriate imaging techniques are selected.

A 13-year-old boy with fever and arthralgia, and an unremarkable family history, was referred to us. His left knee and ankle joints were tender and swollen. No tenderness was observed on the entheses of his spine and right tibial tuberosity. Schober test was normal. Laboratory findings revealed elevated levels of erythrocyte sedimentation rate (68 $\mathrm{mm} / \mathrm{h})$, serum C-reactive protein $(8.8 \mathrm{mg} / \mathrm{dl})$, and matrix metalloprotease-3 (317.3 ng/ml). Both antinuclear antibody and rheumatoid factor were negative. Magnetic resonance imaging (MRI) of his left knee revealed synovitis with massive synovial fluid. Use of $18 \mathrm{~F}$-fluorodeoxyglucose positron emission tomography (FDG-PET) revealed
A

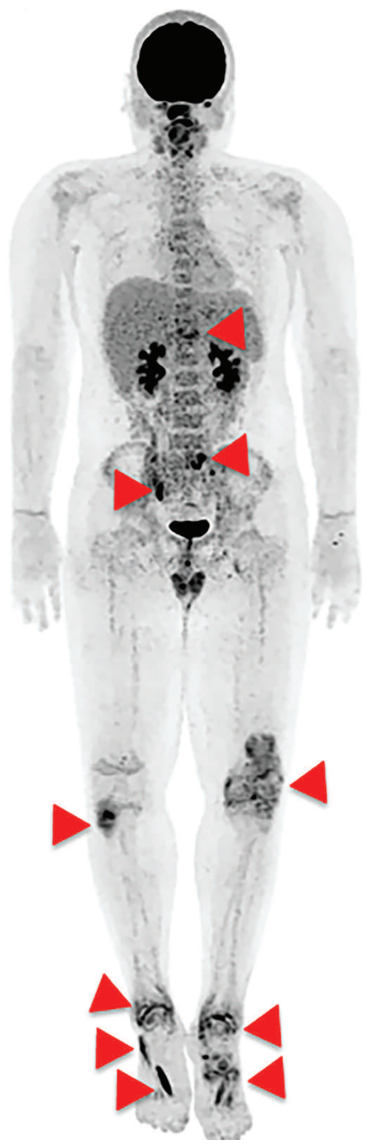

B

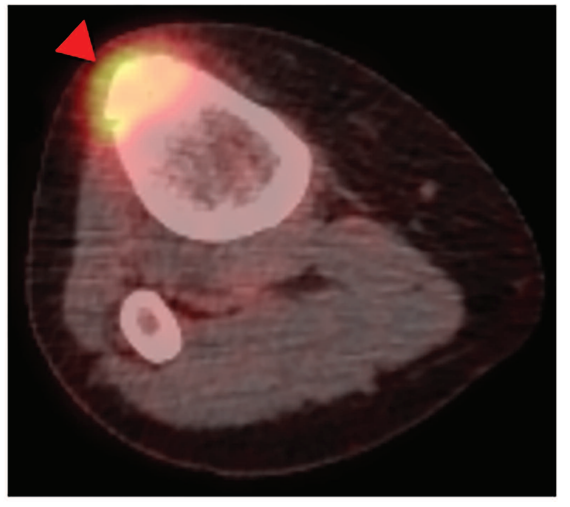

\section{C}

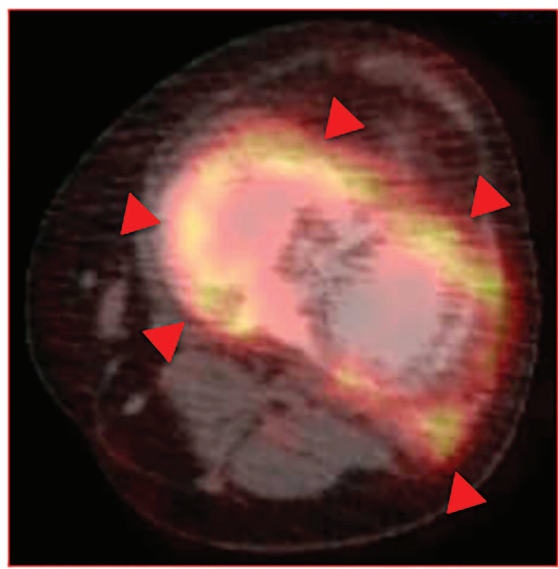

Figure 1. FDG-PET/CT findings. (A) FDG-PET revealed subclinical lesions in the right tibia, thoracic and lumbar vertebrae, right sacroiliac joint, right ankle, and plantar aponeurosis. (B, C) FDG accumulation mainly occurred on the enthesis portion of the ligaments. (B) Right tibial tuberosity. (C) Left knee. FDG-PET: 18F-fluorodeoxyglucose positron emission tomography/computed tomography. 
subclinical lesions in the right tibia, thoracic and lumbar vertebrae, right sacroiliac joint, right ankle, and plantar aponeurosis (Figure 1A). FDG-PET/computed tomography (FDG-PET/CT) revealed FDG accumulation, occurring mainly on the enthesis portion (Figures 1B and 1C). MRI of his pelvis showed right sacroiliitis. ERA was diagnosed. HLA-B27 was positive. Although treatment with ibuprofen was not effective, addition of etanercept alleviated his symptoms.

In our present patient, FDG-PET/CT revealed FDG accumulation in the enthesis rather than in the synovium. FDG-PET has the ability to detect subclinical lesions. Further, the ability to perform FDG-PET for the whole body in 1 scan offers a considerable clinical advantage for extensive assessment of enthesitis. FDG-PET/CT will likely contribute to the ERA diagnosis.

\section{REFERENCE}

1. Aggarwal A, Misra DP. Enthesitis-related arthritis. Clin Rheumatol 2015;34:1839-46. 